EPJ Web of Conferences 58, 01001 (2013)

DOI: $10.1051 /$ epjconf/20135801001

(C) Owned by the authors, published by EDP Sciences, 2013

\title{
Cosmic Time Machines: the Causality Issue
}

\author{
Fernando de Felice ${ }^{1,2, a}$ \\ ${ }^{1}$ Dipartimento di Fisica e Astronomia, Università di Padova, I-35131 Padova, Italy \\ ${ }^{2}$ I.N.F.N. Istituto Nazionale di Fisica Nucleare, Sezione di Padova
}

\begin{abstract}
Continued gravitational collapse gives rise to curvature singularities. If a curvature singularity is globally naked then the space-time may be causally future illbehaved admitting closed time-like or null curves which extend to asymptotic distances and generate a Cosmic Time Machine (de Felice (1995) Lecture Notes in Physics 455, 99 [6]). The existence of Cosmic Time Machines makes it plausible the violation of causality. I conjecture that this circumstance is prevented by some, yet unknown, physical process and show that such a mechanism indeed exists in the Kerr spacetime.
\end{abstract}

\section{Introduction}

If a Naked singularity occurs as result of continued gravitational collapse and is such that it becomes a strong curvature singularity sufficiently far to the future, then a theorem due to Clarke and de Felice (1984) [3] states that strong causality (Chronology) is violated nearby null infinity. Unless prevented by a physical process, such a set would make the entire spacetime causally ill-behaved, giving rise to a Cosmic Time Machine. A Cosmic Time Machine is a space-time which is asymptotically flat and admits closed non-spacelike curves which extend to future infinity. Time machines mix up future and past, memories and expectations, old and young, conclusions and forewords. They challenge human mind making physically legitimate a state of confusion.

Here I shall first recall the properties of a naked singularity and in particular those of a spinning one then will illustrate a physical process which, in a particular circumstance, prevents causality violation in the spacetime of a Kerr naked singularity.

\section{Naked Singularities}

A naked singularity is the outcome of a continued gravitational collapse when no event horizon forms hiding the singularity to the asymptotic region. The properties of a Kerr naked singularity have been extensively investigated in the late seventies ([2];[4]) hence I will recall them briefly. In Boyer and Lindquist coordinates, Kerr metric reads:

$$
\begin{aligned}
d s^{2} & =-\left(1-\frac{2 M r}{\Sigma}\right) d t^{2}-\frac{4 M a r \sin ^{2} \theta}{\Sigma} d t d \phi+\frac{A}{\Sigma} \sin ^{2} \theta d \phi^{2} \\
& +\frac{\Sigma}{\Delta} d r^{2}+\Sigma d \theta^{2}
\end{aligned}
$$

\footnotetext{
ae-mail: defelice@pd.infn.it
} 
where $M$ is the mass of the metric source, $a$ its specific angular momentum ${ }^{1}$ and the functions $\Delta, \Sigma$ and $A$ are given by:

$$
\begin{aligned}
& \Delta=r^{2}-2 M r+a^{2} \\
& \Sigma=r^{2}+a^{2} \cos ^{2} \theta \\
& A=\left(r^{2}+a^{2}\right)^{2}-a^{2} \Delta \sin ^{2} \theta .
\end{aligned}
$$

A time-like geodesic is described by its tangent vector components:

$$
\begin{aligned}
& \dot{t}=(\Delta \Sigma)^{-1}(\text { A }-2 \text { Mar } \ell) \\
& \dot{\theta}= \pm \Sigma^{-1}\left[L+a^{2} \Gamma^{2} \cos ^{2} \theta-\frac{\ell^{2}}{\sin ^{2} \theta}\right]^{1 / 2} \\
& \dot{\phi}=(\Delta \Sigma)^{-1}\left[2 M a \gamma r+\frac{\ell}{\sin ^{2} \theta}(\Sigma-2 M r)\right] \\
& \dot{r}= \pm \Sigma^{-1}\left[(2 M \gamma r-a \ell)^{2}+\Delta\left(r^{2} \Gamma+2 M r \gamma^{2}-L\right)\right]^{1 / 2}
\end{aligned}
$$

where $\Gamma=\gamma^{2}-1$ and a dot means derivative with respect to a real affine parameter along the orbit and $L, \ell$ and $\gamma$ are constants of the motion; the parameter $L$ arises from the separability of the HamiltonJacobi equation in the metric (1) and is related in a non trivial way to (the square of) the total angular momentum of the particle ([5];[8]; [7]), the parameter $\ell$ is the particle's azimuthal angular momentum per unit mass and $\gamma$ is the particle's total energy per unit mass.

In the Kerr spacetime the region where chronology can be violated lies in the $r$-negative sheet of the metric; therefore we restrict ourselves to the family of the vortical geodesics because they are the only ones that can be extended to the $r$-negative sheet and possibly cross the Chronology violation region. The conditions for vortical motion are

$$
\begin{array}{lll}
\Gamma>0, & L<\ell^{2} \leq \frac{\left(L+a^{2} \Gamma\right)^{2}}{4 a^{2} \Gamma}, & a^{2} \Gamma>|L| \\
\Gamma>0, & L=\ell=0 .
\end{array}
$$

\section{The chronology violation set}

Kerr metric admits non trivial chronology violation where the axial Killing vector $\xi_{\phi}^{i}=\delta_{\phi}^{i}$ becomes timelike, that is where

$$
\xi_{\phi}^{i} \xi_{\phi i}=(A / \Sigma) \sin ^{2} \theta<0 .
$$

Except the obvious case of the axis, the above condition is satisfied where $A<0$ and that defines a small region in the $r$-negative part of the metric. The surface equation of this region is given by $A=0$, namely

$$
\Phi_{\phi}^{2} \equiv \sin ^{2} \theta=\frac{\left(r^{2}+a^{2}\right)^{2}}{a^{2} \Delta} \quad r<0
$$

with the obvious condition $0 \leq \Phi_{\phi}^{2} \leq 1$.

\footnotetext{
${ }^{1}$ I use geometrized units, i.e. $c=1=G, c$ and $G$ being respectively the velocity of light in the vacuum and the gravitational constant.
} 
Condition (11) is necessary but not sufficient for chronology violation by a generic time-like world-line. In order to that to occur it is also necessary that the component $\dot{t}$ of the geodesic becomes negative $\dot{t}<0$ hence the chronology set depends on the particular motion we consider. From the geodesic equations we deduce its shape as

$$
\Phi_{c v}^{2}=\Phi_{\phi}^{2}-\frac{2 M r}{a \Delta} \frac{\ell}{\gamma}, \quad r<0 .
$$

It is easy to recognize that the chronology violating set is always contained inside the set bounded by $\Phi_{\phi}^{2}$.

\section{The $\theta=$ constant case}

Let us consider for simplicity the vortical geodesics that are confined on the hyperboloids $\theta=$ constant. The conditions for such a motion can be equivalently written as

$$
\begin{aligned}
L & =-\Gamma a^{2} \cos 2 \theta \\
\ell & =a \sqrt{\Gamma} \sin ^{2} \theta .
\end{aligned}
$$

Equation (13) associates to each vortical geodesic a well defined cv-region. We need to identify for each $\Gamma$ the range of $\theta$ where the above geodesics cross their cv-region. This is done eliminating $\ell$ between (13) and (15) to obtain

$$
\Phi^{2}(\Gamma)=\frac{G\left(r^{2}+a^{2}\right)^{2}}{a^{2}(G \Delta+2 M r)}
$$

where $G=[(\Gamma+1) / \Gamma]^{1 / 2}$. As stated, equation (16) gives, for each $\Gamma$ and for each $\theta$, namely for each vortical geodesic, the intersection with its cv-region.

To complete the answer to the question whether these geodesics really pass through their cvregion, we need to study the locus of their turning points. Equating to zero the component $\dot{r}$ of the geodesic equations and imposing the first of the conditions (14) we obtain the following equation in $\theta$ :

$$
\Phi_{r_{\epsilon}}^{2} \equiv \sin ^{2} \theta_{\epsilon}=\frac{1}{a^{2}}\left[\Delta+2 M r G+\epsilon(G-1)(-2 M r \Delta)^{1 / 2}\right]
$$

with $\epsilon= \pm 1, \quad r<0$. Since $G>1$ the only admissible solution is for $\epsilon=-1$.

Equation (17) depends only on the particle energy $\Gamma$ so together with (16) we can compare, for each $\Gamma$ the relative position of the orbital turning points with the points where the $\theta=$ constant trajectories cross their cv-region. It is rather simple to deduce that it is always ([1])

$$
\Phi^{2}(\Gamma)>\Phi_{r_{-}}^{2} .
$$

This result says that no time-like particle moving freely on the hyperboloid $\theta=$ constant succeeds to reach its cv-region and therefore becoming potentially able to violate causality.

\section{Conclusions}

If naked singularities exist in the Cosmos as predicted by the theory of general relativity then they may give rise to a Cosmic Time Machine. In this case, in fact, the singularity could likely be surrounded by a spacetime region where the local causal future is time-inverted with respect to infinity. As for the energy conservation also the non violation of causality is a fondamental law of physics. Kerr metric shows explicitly such a mechanism which prevents time-like geodesics to enter a state of chronology 
violation and then be in the condition to violate causality. This is a remarkable example of selfconsistency of the solution. In this note we have illustrated this process but unfortunately is not sufficiently general to assure its occurrence for all the other types of orbits. Nevertheless, the actual chronology violation by light-like and accelerated particles does not necessarily imply that they violate causality. There can still be the possibility that Nature provides a mechanism which saves causality even if we do not recognize it, yet.

\section{References}

[1] M. Calvani,F. de Felice, B. Muchotrzeb, and F. Salmistraro Gen. Rel.E Grav. 9 (1978) 155

[2] M. Calvani and F. de Felice Gen. Rel. E Grav. 9 (1978) 889

[3] C.J.S. Clarke and F. de Felice Gen. Rel. E Grav. 16 (1984 )139

[4] F. de Felice and M. Calvani Gen. Rel. E Grav. 4 (1979) 335

[5] F. de Felice J. Phys.A: Math. E Gen. 13 (1980) 1701

[6] F. de Felice Lecture Notes in Physics (Springer Verlag) 455 (1995) 99

[7] F. de Felice and G. Preti Class. Quantum Grav. 16 (1999) 2929

[8] A. Qadir, J. Quamar and M. Rafique Phys. Lett. A109 (1985) 90 